\title{
3D-Printed Cross-Flow Mixer Gradient within Minutes for Microfluidic Applications
}

\author{
Shilpa Sivashankar ${ }^{1}$ \\ ${ }^{1}$ Joint Department of Biomedical Engineering UNC/NCSU \\ Raleigh, North Carolina \\ ssivash@ncsu.edu \\ Hend Mkaouar ${ }^{2}$, Yousof Mashraei ${ }^{2}$, Kholod Alamoudi ${ }^{3}$, \\ Niveen M. Khashab ${ }^{3}$, Khaled Nabil Salama ${ }^{2}$
}

\author{
${ }^{2}$ Computer, Electrical and Mathematical Science and \\ Engineering Division (CEMSE), \\ ${ }^{3}$ Smart Hybrid Materials Laboratory (SHMs), Advanced \\ Membranes and Porous Materials Center, \\ King Abdullah University of Science and Technology \\ (KAUST), Thuwal 23955-6900, Saudi Arabia
}

\begin{abstract}
This paper reports on a compact new cross-flow 3D mixer that is integrated with a gradient generator into one device for toxicology applications. The device has two parts: the first mixes two solvents while the second generates gradients of the obtained solutions. The outlet of the 3D cross-flow mixer is integrated with a linear channel that aids in achieving this gradient by changing flow rates. The dye-visualization test confirm the functionality of mixer and gradient. The mesh structure of the mixer provided excellent mixing regime and is confirmed by experimental and simulation results. The compact size $(25 \times 25 \times 3 \mathrm{~mm})$ and the reduced cost $(\$ 1.5)$ of the device enable the device to be disposable. We aim to study doxorubicin drug at different concentration generated by the device to culture human embryonic kidney (HEK) from the 293-cell line enabling the devices to be used for cellular studies. The carefully designed geometry of the device finds applications in drug toxicology testing devices, micro-total analysis systems ( $\mu$-TAS), and other lab-on-chip devices.
\end{abstract}

Index Terms-Microfluidics; micromixer; 3D printing; crossflow mixer; gradient generator

\section{INTRODUCTION}

In the past two decades, microfluidics has advanced enormously in the field of Bio-MEMS (Bio-Micro-ElectroMechanical Systems) thanks to the development of devices for lab-on-chip (LOC) and micro-total analysis systems. BioMEMS and LOC devices are used to obtain a wide range of measurements including $\mathrm{pH}$, enzyme-reaction kinetics, fluid viscosity, and molecular-diffusion coefficients [1]. Amongst BioMEMS and LOC devices, microfluidic mixers and gradient generators have facilitated significant progress in drug testing and delivery applications [2]. Mixing at microscales is difficult due to low Reynolds numbers and resulting laminar flow [3]; turbulence [4] is hard to obtain at minuscule scales. Because of diffusion, splitting, and recombination channels, good performance is ensured only when a slow flow rate is set at microscale. Hence, a better approach to effective mixing is to increase the interfacial area[5] of contact of fluids by increasing the ratio of surface area to volume. This helps to enhance mixing efficiency by increasing molecule diffusion.

The minuscule channels aid in reducing the time required to transport specific doses of drugs to the cells/ tissues. Also, gradient generators make it possible for chemical species/concentrations to be integrated and manipulated on a single platform. Though there are many gradient generators [6], most are not suitable for all biological applications, as the final concentrations do not depend on the flow rate of the fluids at the inlets [7]. In addition, complex designs are needed to obtain a stabilized and good gradient.

To overcome the above-mentioned limitations, in this paper, we demonstrate a two-layered 3D split and recombine mixer integrated with a linear gradient fabricated via 3D printing technology. The meshed structure of the mixer increases the surface area to volume ratio providing better mixing regimes. The gradients in the device were varied by tuning the flow rates without any complex process. The device was assessed using a dyes-visualization test. An illustration of the integrated device with its application for study the effect of drug doxorubicin at different concentration is represented in Figure 1.

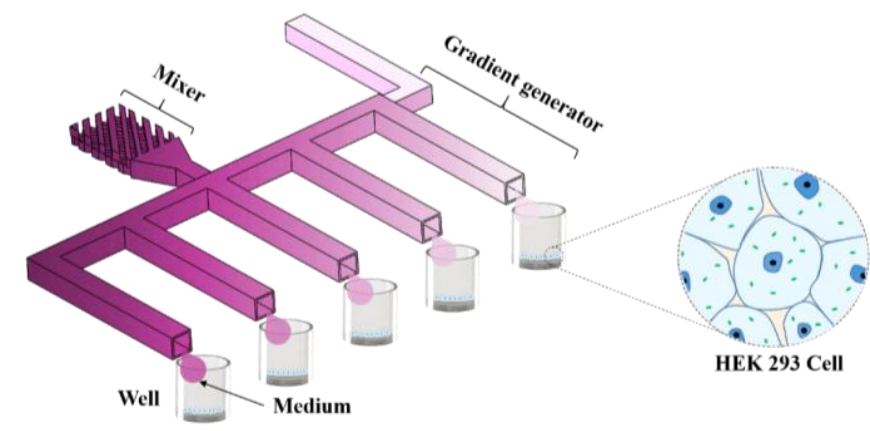

Figure 1. Illustration of the cross-flow mixer gradient

\section{EXPERIMENTAL}

A. Design

The design of the 3D cross-flow mixer-gradient illustrated in Figure 2 allows for efficient mixing schemes within the device by increasing the surface-area-to-volume ratio. As shown in Figure 2(a), the model exhibits a double-layer structure that allows the liquids to be well mixed. The top layer of the mixer consists of two inlet holes for the mixer, one inlet hole for the linear channel, five outlet holes, and a right arm of the mixer channels sloped $50^{\circ}$ from the horizontal plane. The bottom layer of the device consists of the left arm of the mixer channel also sloped at $50^{\circ}$, and a linear channel 
(to act as a gradient generator). It is worth noting that the fluids flow through these slant channels and these are not grooves. These two layers are assembled in Solidworks and printed from one device to form the $3 \mathrm{D}$ cross-flow mixer gradient integrator shown in Figure 2(b).

The printed device is $25 \mathrm{~mm}$ x $25 \mathrm{~mm}$ with a $3 \mathrm{~mm}$ thickness. The holes for the three inlets and the five outlets have the same diameter of $1.2 \mathrm{~mm}$. The length of the five outlet channels for the gradient section measures $4 \mathrm{~mm}$ and the width measures $0.6 \mathrm{~mm}$, while the slant channels for the mixer measure $3 \mathrm{~mm}$ with width $1.5 \mathrm{~mm}$. The depth of all channels is $0.6 \mathrm{~mm}$. As shown in Figure 2(c), the device has three inlets: two mixing inlets and the gradient inlet. The mixer has a fixed input area of $5.3 \mathrm{~mm} \times 3.4 \mathrm{~mm}$ with $4.9 \mathrm{~mm}$ long slant arms. The mixer and gradient inlets are $7.3 \mathrm{~mm}$ apart, and the gradient channel is integrated with the mixer at a distance of $10.2 \mathrm{~mm}$ from the inlet. The mixing chamber is $5.3 \mathrm{~mm}$ long, and the integration chamber is $2.1 \mathrm{~mm}$ across. The length of the gradient channels from outlet $1-5$ is $18 \mathrm{~mm}$ and the length from inlet 3 to the outlet 5 is $10 \mathrm{~mm}$

The device consists of two layers. The fluid flowing in the top and the bottom channel intersect at the mixing region and throughout the mesh structure, the fluids from the top channel traverse to the bottom channel and vice versa. The pattern of the fluid flows was repeated until $5.3 \mathrm{~mm}$ allow mixing of fluids and enable exchange of solutes (dyes) in this mesh structure. The combining region is $2.1 \mathrm{~mm}$ in length and brings in the fluids from both (top and bottom) the channels and enhances exchanges of molecules between the two fluids (blue and yellow dye) injected at the inlets giving exactly the mixed color (green). The fluids will partially be mixed in the mesh structures and is well-mixed in the combining region yielding $50 \%$ of solvent (dyed water). However, the mesh structure allows the fluids to traverse from the top and bottom channel and hence aids in improve mixing of solvents (water). Therefore, both the mixing and combining region are important for this mixer. The gradients generated can be easily tweaked by changing the flowrate at the gradient inlet

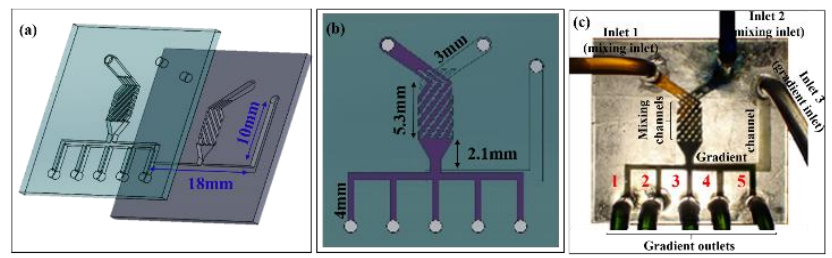

Figure 2: Schematic illustration of the device. (a) Top and bottom layers of the device with gradient generator dimensions. (b) Two layers assembled with the mixer dimensions. (c) Mixing scheme showing the features of the device

\section{B. Simulation}

Simulations were performed with COMSOL Multiphysics commercial software (COMSOL 5.2) to quantify the mixing performance of the micromixer and gradient generator. The mixing and gradient profiles within the unit were investigated using 3D models, as depicted in Figure 3(a). In the simulation models, the type of the fluid used was an incompressible
Newton fluid governed by the Navier-Stokes equation [8]. The component of the fluid is water with a kinematic viscosity of $\mathrm{v}=1 \times 10^{-6} \mathrm{~m}^{2} / \mathrm{s}$ at room temperature. The concentrations of the two different fluids to be mixed were set at $\mathrm{C}=0 \mathrm{~mol} / \mathrm{m}^{3}$ and $\mathrm{C}=100 \mathrm{~mol} / \mathrm{m}^{3}$ at inlets 1 and 2 , respectively. The channel design is identical to the fabricated mixer. The concentration of the input specimen used is $100 \mathrm{~mol} / \mathrm{m}^{3}$, and the liquid medium was water. The result of mixing concentrations is shown in Figure 3 (b). The gradient along channel varies from $69 \%$ to $13 \%$.

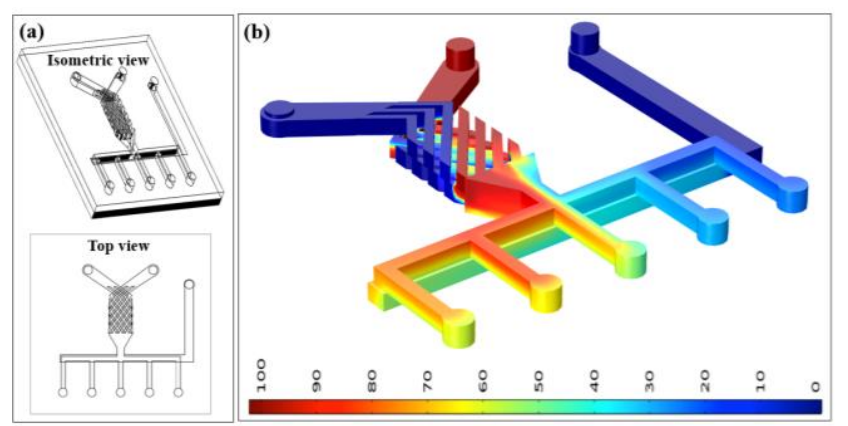

Figure 3. (a) 3D model of the device. (b) Simulation of the device at $1 \mu \mathrm{l} / \mathrm{min}$.

\section{Fabrication of the $3 D$ crss-flow mixer gradient}

The device was fabricated using the MiiCraft+ 3D printer. The $\mathrm{BV}-003$ resin from MiiCraft, which has a viscosity of $150 \mathrm{cps}$ at $28{ }^{\circ} \mathrm{C}$ and a transparent appearance, was used to print the 3D mixer gradient device. The whole device was built by slicing the STL to 56 slices. The time of exposure for the first three slices was set at 20 seconds to ensure adhesion of the device to the picker. For the rest of the 53 slices, the time of exposure was 7 seconds with a layer thickness of 50 microns. The whole fabrication process took about $15 \mathrm{~min}$. The printed device was cleaned immediately by flushing with IPA to remove any unpolymerized resin and then sucked with a vacuum to open the channels, to thereby avoid clogging of the microchannels. To ensure that there is no resin residue, we post-cured the device for five minutes using the post-curing chamber of the MiiCraft+.

\section{Experimental calculation method}

Mixing within the chamber and gradients along the channel depend on mixing between the inlet fluids; hence, we calculated the mixing efficiency by referring to previously published papers $[9,10]$. We calculated the mixing efficiency for 5 points within the mixing chamber and 10 points at the linear channel to determine the gradient profile produced. An Olympus Stylus digital camera was used to capture images of 3D structures. The camera captures many photos in one shot, layer by layer, at different depths. It then overlaps the images to obtain one focused image. Using ImageJ software, the histogram matrix of the selected pixels in the image was extracted at ten different points along the gradient channel. About 200 pixels were selected to obtain the histogram matrix. The histogram matrix corresponds to the pixel intensity $\left(I_{i}\right)$ to 
calculate the total deviation $\left(\sigma_{i}\right)$ at pixel $i$ while calculating the mixing efficiency $(\sigma)$. The dye diffuses along the channel with increasing flow rate and provides the gradient on the linear channel.

The pixel intensities/histogram matrix obtained via image $\mathrm{J}$ at 10 points along the channel is incorporated as $\left(I_{i}\right)$ :

$$
\sigma_{i}=\frac{\left(I_{i}-I_{\text {mix }}\right)}{\left(I_{\text {unmix }}-I_{\text {mix }}\right)}
$$

where $I_{m i x}$ and $I_{\text {unmix }}$ refer to the mean intensity values for 200 pixels at point number 1 and at the inlet of the gradient, respectively. Subsequently, to calculate the gradient along the channel, we calculated the mixing efficiency $(\sigma)$ at 10 points, using:

$$
\sigma=1-\sqrt{\frac{1}{N} \sum_{i=1}^{N}\left(\sigma_{i}\right)^{2}}
$$

\section{RESULTS AND DISCUSSION}

The intricate design of the mixer allows the fluids to be in contact over and over again due to the overlapping structures. The mesh structures of the mixers enables the fluids to experience chaotic flow within the mixer, leading to efficient mixing regimes. The streamlined profile of fluid being mixed is shown via simulation. The flow profile within the mixer (simulated at $500 \mu \mathrm{l} / \mathrm{min}$ ) is represented in Figure 4(a). An enlarged side view of the mixing regimens in the mixer region is showing chaotic waves is shown as subfigure. The flow profile shows that chaotic flow exists and aids in the fluid mixing process in the overlapping structures. Mixing progress along the length of the mixer channel up to region $A$ and it is complete at region $B$, as represented in Figure $4(\mathbf{b})$. The mixing efficiency within the mixer chamber and connecting chamber was plotted in Figure 4(c). Subfigures of the regions chosen for determining the mixing efficiency in both chambers are shown in the same plot. The results reveal that mixing is gradually increasing in the mixing chamber and is complete in the connecting chamber. The initial point of the connecting chamber shows less efficiency due to light variation of the captured image. However it is evident from the experimental results that two different dyes (blue and yellow) mix gradually and form one color (green) at the final stage. We have shown side view of turbulence generated in the beginning section of the device in Fig. 4(a). The whole mixing or the interaction section of the mixer and the fluid flow inside the region is shown in 4(a). Although the mixing efficiency in that region A was progressing along the mesh structures [figure 4(c) inset A] we wanted to achieve complete mix of the fluids and hence, we introduced the combining region [figure $4(\mathrm{c})$ inset $\mathrm{B}$ ] where the mixing efficiency was maximum at the end of the channel. Although there are few mixers that represent mixing efficiency or mixing score, [1113] similar to the one demonstrated in the proposed device, we also produce gradients of the fluid after being mixed which certainly is an advantage for toxicity studies to combine drugs efficiently in the media.

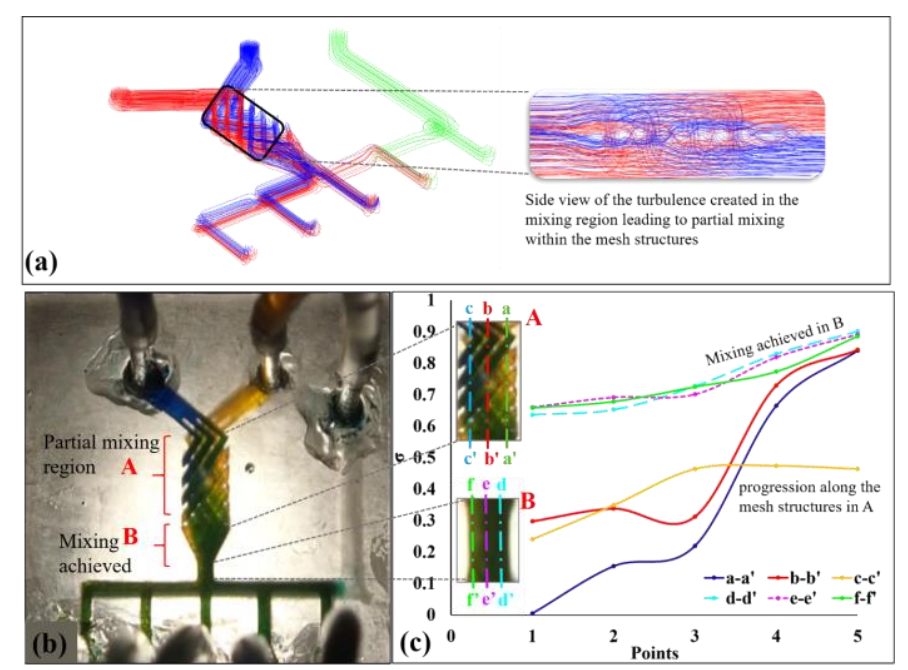

Figure 4: Mixing efficiency in the chamber. (a) Stream and enlarged side-view profile of the mixing unit. An enlarged view of the area selected is shown as a subfigure. (b) Mixing within the chamber with various mixing regions are shown (c) the mixing efficiency within the mixing chamber and connecting chamber [chamber that connects mixer and gradient] is plotted. [Subfigure shows the region chosen for determining mixing scheme in mixing and connecting chamber. a-a', bb', c-c' in mixing chamber and d-d', e-e', f-f' in connecting chamber were the lines that were chosen to plot the mixing efficiency].

The presented gradient generator has many merits over complex gradient generators. When fed at different flow rates, the linear channel has the ability to produce different concentration gradients. The flow rate of the gradient generator varies from $100 \mu \mathrm{l} / \mathrm{min}$ to $500 \mu \mathrm{l} / \mathrm{min}(100,200$, $300,400$, and $500 \mu 1 / \mathrm{min})$. Experiments were carried out for various flow rates of the gradient generator, keeping the inlets of the mixer at a constant flow rate of $500 \mu \mathrm{l} / \mathrm{min}$. The experimental images for different flow rates are shown in Figure 5(a).

The concentration of the output fluid from mixer was diluted with the water fed from the gradient generator. The difference of colors in the five output channels shows the gradient profile that is stable for long term. Figure $\mathbf{5}(\mathbf{b})$ is the normalized concentration values, obtained by the simulating the gradient channel at various flow rates. The mixing efficiency $(\sigma)$ of the fluids at 10 points along the channel is plotted in Figure 5(c).The experimental values are in acceptable agreement with the simulation results. The remaining differences can be explained by the ideal conditions set while simulating. In addition, the simulation values are obtained from whole gradient channels. 


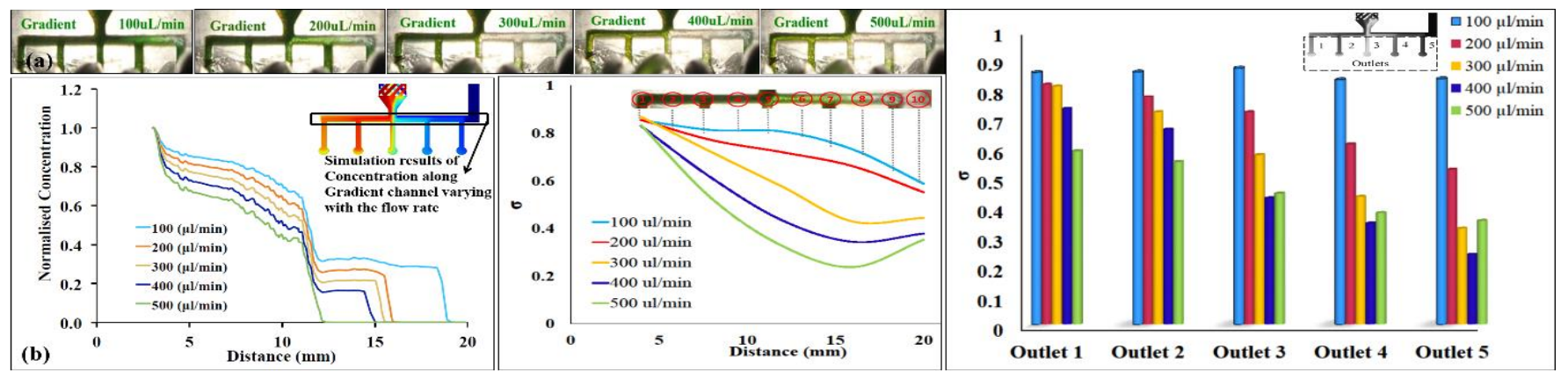

Figure 5: Gradients changing with flow rates. (a) Gradient varying with flow rate is observed (5 panels for five flow rates are shown) (b) The concentration of the gradient channel for various flow rates obtained via simulation. (c) Mixing efficiency calculated along the gradient channel. The points chosen along the channel are shown as a sub figure (d) mixing efficiency at the outlets for varying flowrates.

The results show that the mixing efficiency is not great and doesn't vary too much at the points 7-9 because of the light intensity of the captured images. The image is bright at the center and dark at the edges this makes it difficult to calculate the exact mixing efficiency along the gradient channel. The light intensities for experimental values are obtained from the images taken from the top view that may increase this variation between the results. One of the reasons that compelled us to use the light under the device was the yellowish and blurry appearance of the cured resin, which was transparent in its native form. The change in color of the device resulted from cleaning the device with IPA. The light under the device aided in retrieving maximum information for image processing using the ImageJ software. The mixing efficiency at the outlets with the change in flow rate is plotted in Figure (d) to show there is certainly a difference in the dilution achieved as the flowrate increases.

One of the benefits of the linear gradient generator is that it allowed the gradient profile to be adjusted by modifying the flow rate and also provided the flexibility to be integrated with any other device. This emphasizes that device can be operated at low and high flow rates.

\section{CONCLUSIONS}

We developed a new low-cost miniature multifunctional device for microfluidic application within minutes, thereby avoiding hours of fabrication in a clean room. We have not only integrated a mixer and gradient generator into one compact chip reducing assay time and consumables but also enhanced the mixing efficiency by designing a new cross-flow mixer structure to intensify chaotic flow in the microchannels. This device may find applications that involve disposable microfluidics due to its low cost. We further want to validate drug by culturing the cells fed with the media generating at various concentrations obtained using the device. Tuning the inlet flow rates of the gradient generator can easily vary the concentration of the outlet fluids. The combinatorial functionality of the device represents the characteristic of lab- on-chip devices. In addition, the good geometry of the device finds application in assays that require both low and high flow rates, such as cellular studies and chemical reactions at the microscale.
[1] D. C. Duffy, H. L. Gillis, J. Lin, N. F. Sheppard, and G. J. Kellogg, "Microfabricated Centrifugal Microfluidic Systems: Characterization and Multiple Enzymatic Assays," Analytical Chemistry, vol. 71, pp. 4669-4678, 1999/10/01 1999.

[2] M. W. Ashraf, S. Tayyaba, and N. Afzulpurkar, "Micro Electromechanical Systems (MEMS) Based Microfluidic Devices for Biomedical Applications," International Journal of Molecular Sciences, vol. 12, pp. 3648-3704, 06/07

[3] H. M. Xia, Z. P. Wang, Y. X. Koh, and K. T. May, "A microfluidic mixer with self-excited 'turbulent' fluid motion for wide viscosity ratio applications," Lab on a Chip, vol. 10, pp. 1712-1716, 2010.

[4] J. B. You, K. Kang, T. T. Tran, H. Park, W. R. Hwang, J. M. Kim, et al., "PDMS-based turbulent microfluidic mixer," Lab on a Chip, vol. 15, pp. 1727-1735, 2015.

[5] F. G. Bessoth, A. J. deMello, and A. Manz, "Microstructure for efficient continuous flow mixing," Analytical Communications, vol. 36, pp. 213-215, 1999.

[6] H. Wang, C.-H. Chen, Z. Xiang, M. Wang, and C. Lee, "A convection-driven long-range linear gradient generator with dynamic control," Lab on a Chip, vol. 15, pp. 1445-1450, 2015.

[7] H. Somaweera, A. Ibraguimov, and D. Pappas, "A review of chemical gradient systems for cell analysis," Analytica Chimica Acta, vol. 907, pp. 7-17, 2/11/ 2016.

[8] H. M. Xia, S. Y. M. Wan, C. Shu, and Y. T. Chew, "Chaotic micromixers using two-layer crossing channels to exhibit fast mixing at low Reynolds numbers," Lab on a Chip, vol. 5, pp. 748$755,2005$.

O. Jännig and N.-T. Nguyen, "A polymeric high-throughput pressure-driven micromixer using a nanoporous membrane," Microfluidics and Nanofluidics, vol. 10, pp. 513-519, 2011.

[10] C. Junghun, K. Jinseok, R. Suk-Kyu, P. Jungyul, J. Yongwon, P. Sewan, et al., "A highly efficient 3D micromixer using soft PDMS bonding," Journal of Micromechanics and Microengineering, vol. 16, p. 1778, 2006.

[11] C. Neils, Z. Tyree, B. Finlayson, and A. Folch, "Combinatorial mixing of microfluidic streams," Lab on a Chip, vol. 4, pp. 342350, 2004.

[12] B. H. Peter, Jr., R. M. David, S. L. Frances, P. G. Joel, R. K. Carolyn, and S. O. Elaine, "A combinatorial approach to microfluidic mixing," Journal of Micromechanics and Microengineering, vol. 18, p. 115019, 2008.

[13] S. Sivashankar, S. Agambayev, Y. Mashraei, E. Q. Li, S. T. Thoroddsen, and K. N. Salama, "A "twisted" microfluidic mixer suitable for a wide range of flow rate applications," Biomicrofluidics, vol. 10, May 2016. 